\title{
ZERO DISTRIBUTION OF SEQUENCES OF CLASSICAL ORTHOGONAL POLYNOMIALS
}

\author{
PLAMEN SIMEONOV
}

Received 28 April 2003

We obtain the zero distribution of sequences of classical orthogonal polynomials associated with Jacobi, Laguerre, and Hermite weights. We show that the limit measure is the extremal measure associated with the corresponding weight.

\section{Introduction}

In this paper, we study the zero distribution of sequences of Jacobi, Laguerre, and Hermite polynomials. Our approach is based on identifying these orthogonal polynomials with certain Fekete polynomials defined below, and using monotonicity properties of the zeros of the polynomials.

Let $E \subset \mathbb{R}$ be a closed set that consists of finitely many intervals. Let $w: E \rightarrow$ $[0, \infty)$ be a weight function such that $w(x)>0, x \in \operatorname{Int}(E)$, and $|x| w(x) \rightarrow 0$ as $|x| \rightarrow \infty, x \in E$, if $E$ is unbounded. Consider the function

$$
V_{n}\left(x_{1}, \ldots, x_{n}\right):=\prod_{1 \leq i<j \leq n}\left[w\left(x_{i}\right) w\left(x_{j}\right)\left|x_{j}-x_{i}\right|\right]
$$

$\left\{x_{i}\right\}_{i=1}^{n} \subset E$. It can be shown that $V_{n}$ attains its maximum for some set $\mathscr{F}_{n}=$ $\left\{x_{i}\right\}_{i=1}^{n} \subset E$ called $n$th weighted Fekete set or simply Fekete set.

We introduce the following notation: if $\mu$ is a measure, its logarithmic potential $U^{\mu}(z)$ is defined by

$$
U^{\mu}(z):=\int \log \frac{1}{|z-t|} d \mu(t)
$$

and if $w$ is a weight as defined above, $\mu_{w}$ denotes the corresponding extremal measure [4], which is the unique measure that minimizes the weighted 
logarithmic energy

$$
I_{w}(\mu):=\iint \log \frac{1}{w(z) w(t)|z-t|} d \mu(z) d \mu(t)
$$

over all probability Borel measures supported on $E$. The support of the measure $\mu_{w}$ will be denoted by $S_{w}$.

The asymptotic distribution of Fekete points is known (see [4, Chapter III, Theorem 1.3]).

THEOREM 1.1. Let $\nu_{\mathscr{F}_{n}}$ be the discrete measure that has mass $1 / n$ at each Fekete point $x_{i} \in \mathscr{F}_{n}$. Then,

$$
v_{\mathscr{F}_{n}} \stackrel{w^{*}}{\longrightarrow} \mu_{w}, \quad n \longrightarrow \infty
$$

that is, $\lim _{n \rightarrow \infty} \nu_{\mathscr{F}_{n}}=\mu_{w}$ in the weak-star topology of measures. Furthermore, if $F_{n}$ is the nth degree monic polynomial with zero set $\mathscr{F}_{n}$,

$$
\lim _{n \rightarrow \infty}\left|F_{n}(z)\right|^{1 / n}=\exp \left(-U^{\mu_{w}}(z)\right)
$$

uniformly on compact subsets of $\mathbb{C} \backslash S_{w}$.

We will assume that $w(x)=0$ when $x \in E \backslash \operatorname{Int}(E)$ and $x$ is finite. This condition implies that every Fekete set $\mathscr{F}_{n} \subset \operatorname{Int}(E)$. Consequently, the partial derivatives of $\log \left(V_{n}^{2}\right)$ vanish at the Fekete points:

$$
\begin{aligned}
\frac{d}{d x_{i}}\left(\log V_{n}^{2}\right) & =2(n-1) \frac{w^{\prime}\left(x_{i}\right)}{w\left(x_{i}\right)}+2 \sum_{k \neq i} \frac{1}{x_{i}-x_{k}} \\
& =2(n-1) \frac{w^{\prime}\left(x_{i}\right)}{w\left(x_{i}\right)}+\frac{F_{n}^{\prime \prime}\left(x_{i}\right)}{F_{n}^{\prime}\left(x_{i}\right)}=0, \quad i=1, \ldots, n .
\end{aligned}
$$

In Section 2, we study the zero distribution of Jacobi polynomials $P_{n}^{\left(\alpha_{n}, \beta_{n}\right)}$ with parameters $\alpha_{n}>0$ and $\beta_{n}>0$ that satisfy $\lim _{n \rightarrow \infty} \alpha_{n} / n=2 \alpha>0$ and $\lim _{n \rightarrow \infty} \beta_{n} / n=$ $2 \beta>0$.

In Section 3, we consider Laguerre polynomials $L_{n}^{\left(\alpha_{n}\right)}$ with parameters $\alpha_{n}>0$ that satisfy $\lim _{n \rightarrow \infty} \alpha_{n}=2 \alpha>0$.

In Section 4 , we obtain the zero distribution of the Hermite polynomials $H_{n}$.

Asymptotics and zero distribution of classical orthogonal polynomials have been studied in $[1,2,3,5]$. Here, we extend these results using a simple method that works for all classical orthogonal polynomials.

\section{Zero distribution of Jacobi polynomials}

The Jacobi weight $w_{\alpha, \beta}(x)$ is defined by

$$
w_{\alpha, \beta}(x)=(1-x)^{\alpha}(1+x)^{\beta}, \quad x \in[-1,1],
$$


with positive $\alpha$ and $\beta$. The corresponding extremal measure is given by [4, Chapter IV, Section 5]

$$
d \mu_{w_{\alpha, \beta}}(t)=\frac{1}{\pi} \frac{(1+\alpha+\beta)}{1-t^{2}}((t-a)(b-t))^{1 / 2} d t, \quad t \in S_{w_{\alpha, \beta}},
$$

with support [4, Chapter IV, Section 1]

$$
S_{w_{\alpha, \beta}}=[a, b]=\left[\theta_{2}^{2}-\theta_{1}^{2}-\triangle^{1 / 2}, \theta_{2}^{2}-\theta_{1}^{2}+\triangle^{1 / 2}\right],
$$

where $\theta_{1}=\alpha /(1+\alpha+\beta), \theta_{2}=\beta /(1+\alpha+\beta)$, and $\Delta=\left(1-\left(\theta_{1}+\theta_{2}\right)^{2}\right)\left(1-\left(\theta_{1}-\right.\right.$ $\left.\left.\theta_{2}\right)^{2}\right)$.

Let $P_{n}^{(\alpha, \beta)}$ and $q_{n, \alpha, \beta}$ denote the orthonormal polynomial of degree $n$ and the monic orthogonal polynomial of degree $n$, respectively, with respect to the weight $w_{\alpha, \beta}$. Let

$$
v_{n, \alpha, \beta}:=\frac{1}{n} \sum_{x: P_{n}^{(\alpha, \beta)}(x)=0} \delta(x)
$$

denote the discrete probability measure with mass $1 / n$ at each zero of $P_{n}^{(\alpha, \beta)}$. Here, $\delta(x)$ denotes the discrete probability measure with support $x$ (the point mass at $x$ ).

We first show that the Fekete polynomials for Jacobi weights $w_{\alpha, \beta}$ with $\alpha>0$ and $\beta>0$ are, in fact, Jacobi polynomials.

Let $\alpha>0$ and $\beta>0$ be fixed and set $w=w_{\alpha, \beta}^{1 /(n-1)}$ in the function $V_{n}$ defined with (1.1). Since

$$
\frac{w^{\prime}(x)}{w(x)}=\frac{1}{(n-1)} \frac{w_{\alpha, \beta}^{\prime}(x)}{w_{\alpha, \beta}(x)}=\frac{1}{(n-1)} \frac{\beta-\alpha-(\alpha+\beta) x}{\left(1-x^{2}\right)}, \quad x \in(-1,1),
$$

equations (1.6) yield

$$
2\left(\beta-\alpha-(\alpha+\beta) x_{i}\right) F_{n}^{\prime}\left(x_{i}\right)+\left(1-x_{i}^{2}\right) F_{n}^{\prime \prime}\left(x_{i}\right)=0, \quad i=1, \ldots, n .
$$

Thus, the polynomial $\left(1-x^{2}\right) F_{n}^{\prime \prime}(x)+2(\beta-\alpha-(\alpha+\beta) x) F_{n}^{\prime}(x)$ of degree $n$ with leading coefficient $-n(n+2 \alpha+2 \beta-1)$ has zeros at $x_{1}, \ldots, x_{n}$, and therefore

$$
\left(1-x^{2}\right) F_{n}^{\prime \prime}(x)+2(\beta-\alpha-(\alpha+\beta) x) F_{n}^{\prime}(x)+n(n+2 \alpha+2 \beta-1) F_{n}(x)=0 .
$$

By [6, Theorem 4.2.1], the polynomial $q_{n, 2 \alpha-1,2 \beta-1}$ satisfies (2.7) as well. However, (2.7) has a unique monic polynomial solution of degree $n$. Indeed, the polynomial

$$
r:=F_{n}-q_{n, 2 \alpha-1,2 \beta-1}=\sum_{j=0}^{n-1} c_{j} q_{j, 2 \alpha-1,2 \beta-1}
$$


satisfies (2.7). Substituting $r$ in (2.7), we obtain

$$
\begin{aligned}
& 0=\left(1-x^{2}\right) r^{\prime \prime}(x)+2(\beta-\alpha-(\alpha+\beta) x) r^{\prime}(x)+n(n+2 \alpha+2 \beta-1) r(x) \\
&= \sum_{j=0}^{n-1} c_{j}\left[\left(1-x^{2}\right) q_{j, 2 \alpha-1,2 \beta-1}^{\prime \prime}(x)+2(\beta-\alpha-(\alpha+\beta) x) q_{j, 2 \alpha-1,2 \beta-1}^{\prime}(x)\right. \\
&\left.\quad \quad+n(n+2 \alpha+2 \beta-1) q_{j, 2 \alpha-1,2 \beta-1}(x)\right] \\
&=\sum_{j=0}^{n-1} c_{j}\left(-j^{2}+n^{2}+(n-j)(2 \alpha+2 \beta-1)\right) q_{j, 2 \alpha-1,2 \beta-1}(x),
\end{aligned}
$$

where (2.7) was applied to $q_{j, 2 \alpha-1,2 \beta-1}, j=0, \ldots, n-1$. Since $(n-j)(n+j+2 \alpha+$ $2 \beta-1)>0$ for $j=0, \ldots, n-1,(2.9)$ implies $c_{j}=0, j=0, \ldots, n-1$, and the uniqueness of the polynomial solution of (2.7) follows.

We have shown that for positive $\alpha$ and $\beta$, the $n$th Fekete polynomial $F_{n, \alpha, \beta}$ associated with the Jacobi weight $w_{\alpha, \beta}$ is the Jacoby polynomial $q_{n, 2(n-1) \alpha-1,2(n-1) \beta-1}$.

TheOrem 2.1. Let $\left\{\alpha_{n}\right\}$ and $\left\{\beta_{n}\right\}$ be sequences of positive numbers satisfying

$$
\frac{\alpha_{n}}{n} \longrightarrow 2 \alpha>0, \quad \frac{\beta_{n}}{n} \longrightarrow 2 \beta>0, \quad n \longrightarrow \infty .
$$

If $\alpha$ and $\beta$ are finite, then

$$
v_{n, \alpha_{n}, \beta_{n}} \stackrel{w^{*}}{\longrightarrow} \mu_{\alpha, \beta}, \quad n \longrightarrow \infty .
$$

If $\alpha=\infty$ and $\beta$ is finite, the limit of the measures $\nu_{n, \alpha_{n}, \beta_{n}}$ is the point mass at -1 . If $\alpha$ is finite and $\beta=\infty$, the limit of the measures $\nu_{n, \alpha_{n}, \beta_{n}}$ is the point mass at 1 .

If $\alpha=\beta=\infty$ and $\alpha_{n} / \beta_{n} \rightarrow \lambda>0$ as $n \rightarrow \infty$, the limit measure is the point mass at $(1-\lambda) /(1+\lambda)$.

Proof. For fixed $\alpha>0$ and $\beta>0$, let $\left\{x_{i, n}^{(\alpha, \beta)}\right\}_{i=1}^{n}$ be the $n$th Fekete set, and let $\nu_{n, \alpha, \beta}$ denote the discrete probability measure having mass $1 / n$ at each Fekete point $x_{i, n}^{(\alpha, \beta)}$. By Theorem 1.1,

$$
v_{n, \alpha, \beta} \stackrel{w^{*}}{\longrightarrow} \mu_{w_{\alpha, \beta},}, \quad n \longrightarrow \infty .
$$

From (2.10), it follows that

$$
\tilde{\alpha}_{n}:=\frac{\alpha_{n}+1}{2(n-1)} \longrightarrow \alpha, \quad \tilde{\beta}_{n}:=\frac{\beta_{n}+1}{2(n-1)} \longrightarrow \beta, \quad n \longrightarrow \infty .
$$

Furthermore,

$$
F_{n, \tilde{\alpha}_{n}, \tilde{\beta}_{n}}=q_{n, \alpha_{n}, \beta_{n}} .
$$

Assume first that $\alpha$ and $\beta$ are both finite. Let $\epsilon>0$ be fixed and let $N(\epsilon)$ be such that $\alpha-\epsilon \leq \tilde{\alpha}_{n} \leq \alpha+\epsilon$ and $\beta-\epsilon \leq \tilde{\beta}_{n} \leq \beta+\epsilon$ for $n \geq N(\epsilon)$. We will use 
a certain monotonicity property of the zeros of the Jacobi polynomials. For $0<$ $\alpha_{1}<\alpha_{2}$ and $0<\beta_{1}<\beta_{2}$,

$$
\frac{w_{\alpha_{1}, \beta}(x)}{w_{\alpha_{2}, \beta}(x)}=(1-x)^{\alpha_{1}-\alpha_{2}}, \quad \frac{w_{\alpha, \beta_{2}}(x)}{w_{a, \beta_{1}}(x)}=(1+x)^{\beta_{2}-\beta_{1}}
$$

are increasing functions on $(-1,1)$. By $[6$, Theorem 6.12.2],

$$
x_{j, n}^{\left(\alpha_{2}, \beta\right)}<x_{j, n}^{\left(\alpha_{1}, \beta\right)}, \quad x_{j, n}^{\left(\alpha, \beta_{1}\right)}<x_{j, n}^{\left(\alpha, \beta_{2}\right)}, \quad j=1, \ldots, n .
$$

Therefore,

$$
x_{j, n}^{(\alpha+\epsilon, \beta-\epsilon)}<x_{j, n}^{\left(\tilde{\alpha}_{n}, \tilde{\beta}_{n}\right)}<x_{j, n}^{(\alpha-\epsilon, \beta+\epsilon)}, \quad j=1, \ldots, n .
$$

Let $A \subset S_{w_{\alpha, \beta}}$ be an interval. We have

$$
\left|v_{n, \tilde{\alpha}_{n}, \tilde{\beta}_{n}}(A)-\mu_{w_{\alpha, \beta}}(A)\right| \leq\left|v_{n, \tilde{\alpha}_{n}, \tilde{\beta}_{n}}(A)-v_{n, \alpha, \beta}(A)\right|+\left|v_{n, \alpha, \beta}(A)-\mu_{w_{\alpha, \beta}}(A)\right| .
$$

In view of (2.12), it is enough to estimate the first term in (2.18). For any measurable set $B$ and fixed $\alpha_{0}>0$ and $\beta_{0}>0$, from (2.2) and (2.12), it follows that

$$
\begin{aligned}
& \left|v_{n, \alpha^{\prime}, \beta^{\prime}}(B)-v_{n, \alpha_{0}, \beta_{0}}(B)\right| \\
& \leq\left|v_{n, \alpha^{\prime}, \beta^{\prime}}(B)-\mu_{w_{\alpha^{\prime}, \beta^{\prime}}}(B)\right|+\left|\mu_{w_{\alpha^{\prime}, \beta^{\prime}}}(B)-\mu_{w_{\alpha_{0}, \beta_{0}}}(B)\right| \\
& \quad+\left|v_{n, \alpha_{0}, \beta_{0}}(B)-\mu_{w_{\alpha_{0}, \beta_{0}}}(B)\right| \longrightarrow 0
\end{aligned}
$$

if we let $n \rightarrow \infty$ first, and then $\alpha^{\prime} \rightarrow \alpha_{0}$ and $\beta^{\prime} \rightarrow \beta_{0}$.

Next, define

$$
J_{n, \alpha, \beta}^{L}(a):=\max \left\{j: x_{j, n}^{(\alpha, \beta)}<a\right\}, \quad J_{n, \alpha, \beta}^{R}(a):=\min \left\{j: x_{j, n}^{(\alpha, \beta)}>a\right\} .
$$

Let $A=[c, d]$. By (2.19),

$$
n^{-1}\left|J_{n, \alpha \pm \epsilon, \beta \pm \epsilon}^{L}(c)-J_{n, \alpha, \beta}^{L}(c)\right|=\left|\left(v_{n, \alpha \pm \epsilon, \beta \pm \epsilon}-v_{n, \alpha, \beta}\right)((-\infty, c))\right| \longrightarrow 0
$$

as $n \rightarrow \infty$ first, and then $\epsilon \rightarrow 0$. Similarly,

$$
n^{-1}\left|J_{n, \alpha \pm \epsilon, \beta \pm \epsilon}^{R}(d)-J_{n, \alpha, \beta}^{R}(d)\right| \longrightarrow 0, \quad n \longrightarrow \infty, \epsilon \longrightarrow 0 .
$$

Furthermore, (2.17) implies

$$
\begin{aligned}
& J_{n, \alpha-\epsilon, \beta+\epsilon}^{L}(c) \leq J_{n, \tilde{\alpha}_{n}, \tilde{\beta}_{n}}^{L}(c) \leq J_{n, \alpha+\epsilon, \beta-\epsilon}^{L}(c), \\
& J_{n, \alpha-\epsilon, \beta+\epsilon}^{R}(d) \leq J_{n, \tilde{\alpha}_{n}, \tilde{\beta}_{n}}^{R}(d) \leq J_{n, \alpha+\epsilon, \beta-\epsilon}^{R}(d) .
\end{aligned}
$$


From (2.21), (2.22), and (2.23) it follows that

$$
\left|v_{n, \tilde{\alpha}_{n}, \tilde{\beta}_{n}}(A)-v_{n, \alpha, \beta}(A)\right| \longrightarrow 0, \quad n \longrightarrow \infty,
$$

and this completes the proof for finite $\alpha$ and $\beta$.

If $\alpha$ is finite and $\beta=\infty, \beta$ is finite and $\alpha=\infty$, or $\alpha$ and $\beta$ are both infinite, and $\alpha_{n} / \beta_{n} \rightarrow \lambda \geq 0$ as $n \rightarrow \infty$, it immediately follows from (2.3) that the supports of the extremal measures $S_{w_{\alpha_{n}, \beta_{n}}}$ shrink to the single point $1,-1$, or $(1-\lambda) /(1+\lambda)$, respectively, which establishes the proof in these cases.

\section{Zero distribution of Laguerre polynomials}

Let $L_{n}^{(\alpha)}(x)$ denote the monic Laguerre polynomials that are orthogonal with respect to the Laguerre weight $w_{\alpha}(x)=x^{\alpha} e^{-x}$ on $[0, \infty)$, when $\alpha>-1$. Furthermore, $y=L_{n}^{(\alpha)}$ is the unique polynomial solution of degree $n$ of the differential equation

$$
x y^{\prime \prime}+(\alpha+1-x) y^{\prime}+n y=0
$$

When $\alpha>0$, the extremal measure $\mu_{w_{\alpha}}$ is given by (see [4, Chapter IV, Section 5])

$$
d \mu_{w_{\alpha}}(t)=\frac{1}{\pi t}\left(\left(t-a_{\alpha}\right)\left(b_{\alpha}-t\right)\right)^{1 / 2} d t, \quad t \in S_{w_{\alpha}}
$$

where (see [4, Chapter IV, Section 1])

$$
S_{w_{\alpha}}=\left[a_{\alpha}, b_{\alpha}\right]=\left[\alpha+1-(2 \alpha+1)^{1 / 2}, \alpha+1+(2 \alpha+1)^{1 / 2}\right] .
$$

To show that the Fekete polynomials for Laguerre weights $w_{\alpha}$ with $\alpha>0$ are Laguerre polynomials, we set $w=w_{\alpha}$ in (1.1). Since $w^{\prime}(x) / w(x)=(\alpha / x-1)$, (1.6) takes the form

$$
x_{i} F_{n}^{\prime \prime}\left(x_{i}\right)+2(n-1)\left(\alpha-x_{i}\right) F_{n}^{\prime}\left(x_{i}\right)=0, \quad i=1, \ldots, n,
$$

where $F_{n}=F_{n, \alpha}$ is the $n$th Fekete polynomial for the weight $w_{\alpha}$. Since $2(n-$ 1) $(\alpha-x) F_{n}^{\prime}(x)+x F_{n}^{\prime \prime}(x)$ is a polynomial of degree $n$ with leading coefficient $-2 n(n-1)$, the above equations imply that $z=F_{n}$ satisfies the differential equation

$$
t z^{\prime \prime}+2(n-1)(\alpha-t) z^{\prime}+2 n(n-1) z=0 .
$$

Setting $z(t)=y(x)$ with $x=\lambda t$, we get $d^{k} z / d t^{k}=\lambda^{k} d^{k} y / d x^{k}$ for every $k \geq 0$, and (3.5) becomes

$$
\lambda x y^{\prime \prime}+2(n-1)(\lambda \alpha-x) y^{\prime}+2 n(n-1) y=0 .
$$


Choosing $\lambda=2(n-1)$, we obtain

$$
x y^{\prime \prime}+(2(n-1) \alpha-x) y^{\prime}+n y=0
$$

From (3.1) and (3.7) it follows that $y(x)=L_{n}^{(2(n-1) \alpha-1)}(x)$. Since $F_{n}(t)=z(t)=$ $y(2(n-1) t)$ we obtain

$$
F_{n, \alpha}(x)=L_{n}^{(2(n-1) \alpha-1)}(2(n-1) x) .
$$

Equation (3.8) shows that for every $n \geq 1$ there is a unique $n$th Fekete set $\left\{x_{i, n}^{(\alpha)}\right\}_{i=1}^{n}$, and if $\left\{z_{i, n}^{(\gamma)}\right\}_{i=1}^{n}$ denotes the zero set of the Laguerre polynomial $L_{n}^{(\gamma)}$ with $\gamma>0$, then

$$
x_{i, n}^{(\alpha)}=\frac{z_{i, n}^{(2(n-1) \alpha-1)}}{2(n-1)}, \quad i=1, \ldots, n,
$$

where both the zeros of the Laguerre polynomial and the Fekete points are arranged in increasing order.

Next, we show that the Fekete sets for a weight $w_{\gamma}$ with $\gamma>0$ are contained in a compact set. By [4, Chapter I, Theorem 1.3],

$$
U^{\mu_{w_{\gamma}}}(x)-\log w_{\gamma}(x)=F_{w_{\gamma}}, \quad x \in S_{w_{\gamma}},
$$

where $F_{w_{y}}$ is a constant. Furthermore, by [7, Theorem A], $U^{\mu_{w_{\gamma}}}(x)-\log w_{\gamma}(x) \geq$ $F_{w_{y}}, x \notin S_{w_{\gamma}}$. This function is then continuously differentiable on $(0, \infty) \backslash\left\{a_{\gamma}\right.$, $\left.b_{\gamma}\right\}$, its first derivative vanishes on $\left(a_{\gamma}, b_{\gamma}\right)$, and

$$
\frac{d^{2}}{d x^{2}}\left(U^{\mu_{w_{\gamma}}}(x)-\log w_{\gamma}(x)\right)=\int_{a_{\gamma}}^{b_{\gamma}} \frac{1}{(x-t)^{2}} d \mu_{w_{\gamma}}(t)+\frac{\gamma}{x^{2}}>0, \quad x>b_{\gamma} .
$$

Thus, the first derivative of $U^{\mu_{w_{\gamma}}}(x)-\log w_{\gamma}(x)$ is positive for $x>b_{\gamma}$, and so $U^{\mu_{w_{\gamma}}}(x)-\log w_{\gamma}(x)>F_{w_{\gamma}}$ for $x>b_{\gamma}$. Therefore,

$$
S_{w_{\gamma}}^{*}:=\left\{x: U^{\mu_{w_{\gamma}}}(x)-\log w_{\gamma}(x) \leq F_{w_{\gamma}}\right\} \subset\left[0, b_{\gamma}\right] .
$$

By [4, Chapter III, Theorem 1.2], $\left\{x_{i, n}^{(\gamma)}\right\}_{i=1}^{n} \subset S_{w_{\gamma}}^{*}$. Thus, we conclude that $\left\{x_{i, n}^{(\gamma)}\right\}_{i=1}^{n}$ $\subset\left[0, b_{\gamma}\right]$ for every $n$.

Theorem 3.1. Let $\left\{\alpha_{n}\right\} \subset(0, \infty)$ be a sequence satisfying $\alpha_{n} / n \rightarrow 2 \alpha>0$ as $n \rightarrow \infty$. Then,

$$
\nu_{n, \alpha_{n}}:=\frac{1}{n} \sum_{i=1}^{n} \delta\left(\frac{z_{i, n}^{\left(\alpha_{n}\right)}}{2(n-1)}\right) \stackrel{w^{*}}{\longrightarrow} \mu_{w_{\alpha}}, \quad n \longrightarrow \infty .
$$


Proof. We have $\tilde{\alpha}_{n}:=\left(\alpha_{n}+1\right) /(2(n-1)) \rightarrow \alpha>0$ as $n \rightarrow \infty$. By (3.9), $z_{i, n}^{\left(\alpha_{n}\right)} /(2(n-$ $1))=x_{i, n}^{\left(\tilde{\alpha}_{n}\right)}, i=1, \ldots, n$, and by [4, Chapter III, Theorem 1.3],

$$
\frac{1}{n} \sum_{i=1}^{n} \delta\left(x_{i, n}^{(\alpha)}\right) \stackrel{w^{*}}{\longrightarrow} \mu_{w_{\alpha}}, \quad n \longrightarrow \infty .
$$

The rest of the proof follows the argument used in the proof of Theorem 2.1. In this case, the zeros of the Laguerre polynomials $L_{n}^{(\alpha)}$ are monotone in the sense that if $\alpha_{1}>\alpha_{2}>-1$, then $z_{i, n}^{\left(\alpha_{2}\right)}<z_{i, n}^{\left(\alpha_{1}\right)}, i=1, \ldots, n$. This follows from the fact that $w_{\alpha_{1}}(x) / w_{\alpha_{2}}(x)=x^{\alpha_{1}-\alpha_{2}}$ is an increasing function on $[0, \infty)$, and a variation of $[6$, Theorem 6.12.2] for unbounded intervals.

\section{Zero distribution of the Hermite polynomials}

The monic Hermite polynomials $H_{n}$ are orthogonal with respect to the weight $w(x)=e^{-x^{2}}, x \in \mathbb{R}$. Furthermore, $y=H_{n}$ satisfies the differential equation

$$
y^{\prime \prime}-2 x y^{\prime}+2 n y=0, \quad n \geq 0 .
$$

The corresponding extremal measure $\mu_{w}$ is given by (see [4, Chapter IV, Theorem 5.1]),

$$
d \mu_{w}(t)=\frac{2}{\pi} \sqrt{1-t^{2}} d t, \quad t \in[-1,1] .
$$

To determine the relationship between the zeros of the Hermite polynomials and the Fekete sets for the weight $w(x)=e^{-x^{2}}$, we set $w(x)=e^{-x^{2}}$ in (1.1). Since $w^{\prime}(x) / w(x)=-2 x,(1.6)$ yields

$$
4(n-1) x_{i} F_{n}^{\prime}\left(x_{i}\right)-F_{n}^{\prime \prime}\left(x_{i}\right)=0, \quad i=1, \ldots, n .
$$

These equations imply that the $n$th degree polynomial $4(n-1) x F_{n}^{\prime}(x)-F_{n}^{\prime \prime}(x)$ with leading coefficient $4 n(n-1)$ has the same zero set as $F_{n}(x)$. Therefore, $F_{n}(x)$ is the polynomial solution of the differential equation

$$
z^{\prime \prime}-4(n-1) x z^{\prime}+4 n(n-1) z=0
$$

For $\lambda>0$, we set $y(x)=z(\lambda x)$. From (4.4), it follows that

$$
y^{\prime \prime}-4(n-1) \lambda^{2} x y^{\prime}+4 \lambda^{2} n(n-1) y=0,
$$

and in particular $y(x)=F_{n}(x / \sqrt{2(n-1)})$ satisfies (4.1). Since (4.1) has a unique polynomial solution of degree $n$, we obtain $F_{n}(x / \sqrt{2(n-1)})=H_{n}(x)$. Then, if $\left\{x_{i, n}\right\}_{i=1}^{n}$ and $\left\{z_{i, n}\right\}_{i=1}^{n}$ denote the zeros of $F_{n}$ and $H_{n}$, respectively, we have

$$
x_{i, n}=\frac{z_{i, n}}{\sqrt{2(n-1)}}, \quad i=1, \ldots, n .
$$


From [4, Chapter IV, equation (5.5)], it follows that $S_{w}^{*}=[-1,1]$, and then by [4, Chapter III, Theorem 1.2], $\left\{x_{i, n}\right\}_{i=1}^{n} \subset[-1,1]$ for every $n \geq 1$. Using the argument employed in the previous sections, we establish the following theorem.

Theorem 4.1. For every $n \geq 1$, let $v_{n}$ denote the discrete probability measure having mass $1 / n$ at each zero $z_{i, n}$ of the Hermite polynomial $H_{n}$. Then,

$$
v_{n}:=\frac{1}{n} \sum_{i=1}^{n} \delta\left(\frac{z_{i, n}}{\sqrt{2(n-1)}}\right) \stackrel{w^{*}}{\longrightarrow} \mu_{w}, \quad n \longrightarrow \infty .
$$

\section{References}

[1] L.-C. Chen and M. E. H. Ismail, On asymptotics of Jacobi polynomials, SIAM J. Math. Anal. 22 (1991), no. 5, 1442-1449.

[2] M. Lachance, E. B. Saff, and R. S. Varga, Bounds for incomplete polynomials vanishing at both endpoints of an interval, Constructive Approaches to Mathematical Models (Proc. Conf. in honor of R. J. Duffin, Pittsburgh, Pa, 1978), Academic Press, New York, 1979, pp. 421-437.

[3] D. S. Moak, E. B. Saff, and R. S. Varga, On the zeros of Jacobi polynomials $P_{n}^{\left(\alpha_{n}, \beta_{n}\right)}(x)$, Trans. Amer. Math. Soc. 249 (1979), no. 1, 159-162.

[4] E. B. Saff and V. Totik, Logarithmic Potentials with External Fields, Grundlehren der mathematischen Wissenschaften, vol. 316, Springer-Verlag, Berlin, 1997.

[5] E. B. Saff, J. L. Ullman, and R. S. Varga, Incomplete polynomials: an electrostatics approach, Approximation Theory, III (Proc. Conf., Univ. Texas, Austin, Tex, 1980), Academic Press, New York, 1980, pp. 769-782.

[6] G. Szego, Orthogonal Polynomials, 4th ed., Colloquium Publications, vol. 23, American Mathematical Society, Rhode Island, 1975.

[7] V. Totik, Weighted Approximation with Varying Weight, Lecture Notes in Mathematics, vol. 1569, Springer-Verlag, Berlin, 1994.

Plamen Simeonov: Department of Computer and Mathematical Sciences, University of Houston-Downtown, Houston, TX 77002, USA

E-mail address: simeonov@uhd.edu 


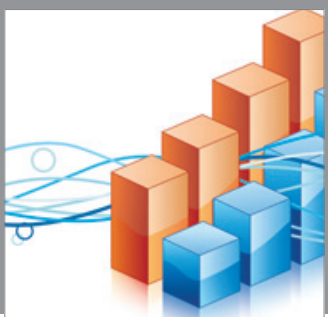

Advances in

Operations Research

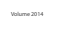

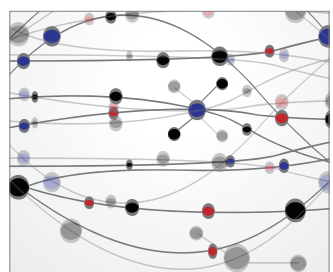

\section{The Scientific} World Journal
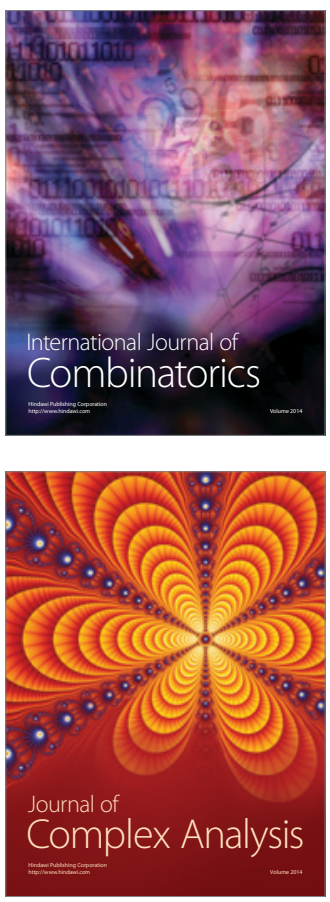

International Journal of

Mathematics and

Mathematical

Sciences
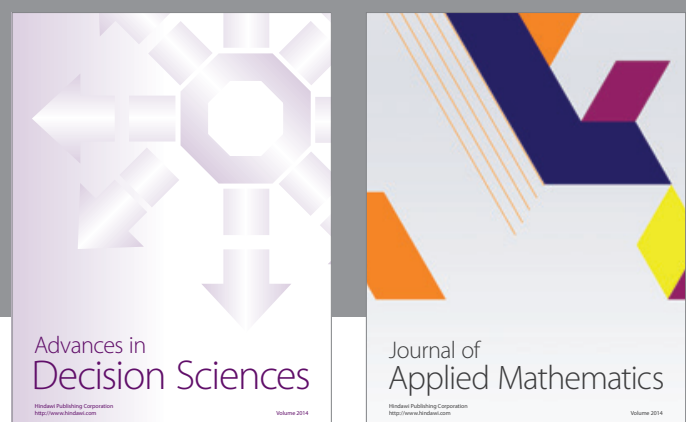

Journal of

Applied Mathematics
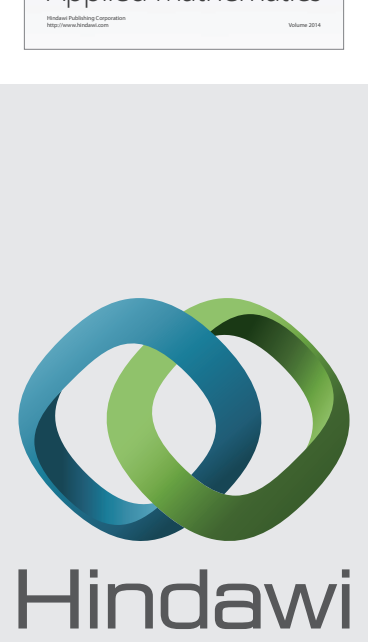

Submit your manuscripts at http://www.hindawi.com
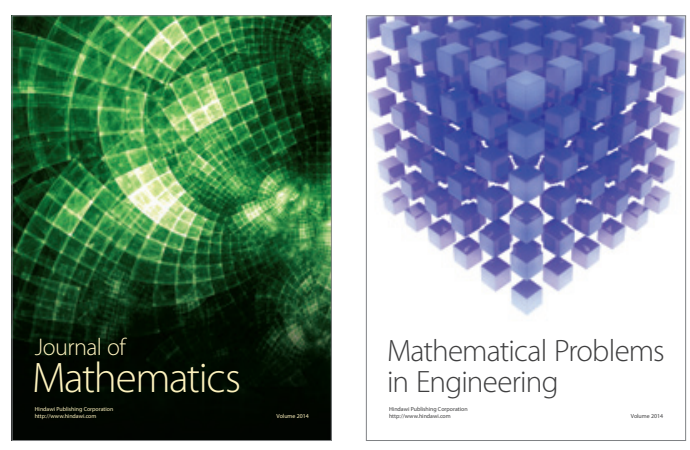

Mathematical Problems in Engineering
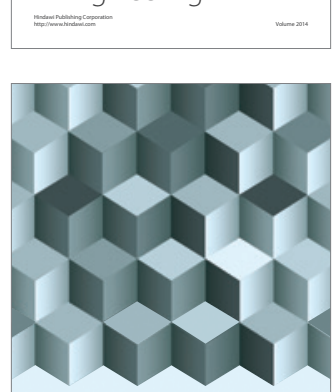

Journal of

Function Spaces
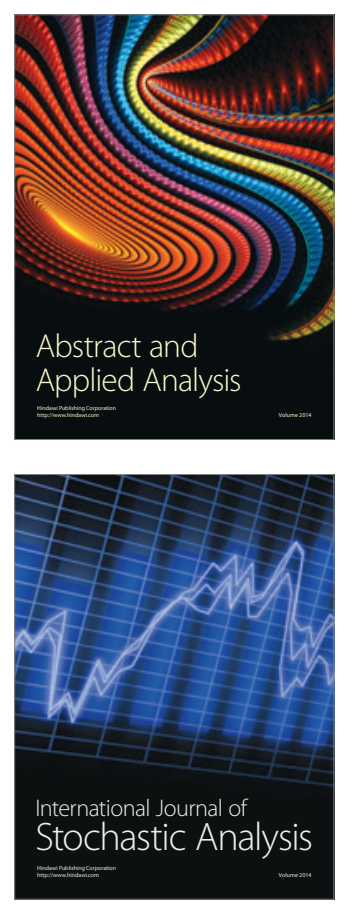

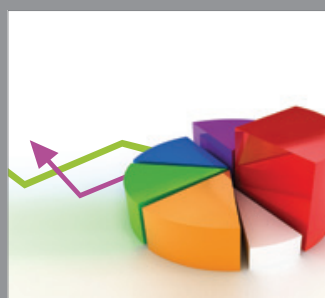

ournal of

Probability and Statistics

Promensencen
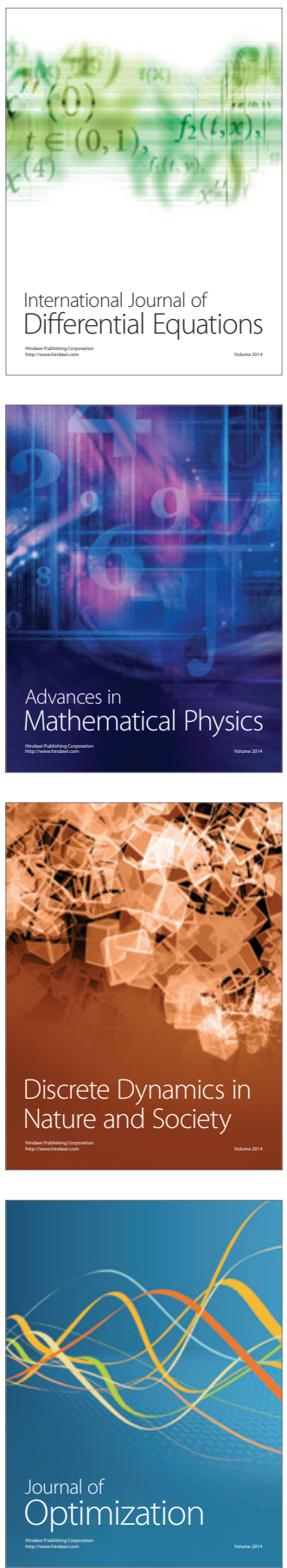